\title{
Bhatia's Battery of Performance Tests of Intelligence: A Critical Appraisal
}

\author{
B.N. Roopesh \\ Neurosciences, Bengaluru. \\ Corresponding author: B.N.Roopesh \\ Email -bn.roopesh@gmail.com
}

Additional Professor, Department of Clinical Psychology, National Institute of Mental Health and

\begin{abstract}
Bhatia's Battery of Performance Tests of Intelligence, is one of the popular tests of intelligence in India. Despite some of its limitations, such as that it has norms only between 69 to 131 IQ points, that it is standardized only on boys who are 11 to 16 years of age and it is developed in 1950s, the battery still is one of the favourites among many psychologists. Some of the positives which psychologists attribute to it are: that it is a performance test and can be easily administered to language incompatible/delayed subject; that it is easy to administer and score; that the test interesting and due to which elicits good cooperation among subjects; and it has separate sets of norms for people who are literate and people who are illiterate. However, rarely it is questioned as to why there should be a separate set of norms for illiterate group? Does having separate set of norms adhere to the standard practices of ability testing or assessment of individual differences? Does having separate set of norms affects the identification of actual and correct intellectual abilities of either illiterate or literate group? and Does the separate set of norms for illiterate affects them adversely? This article provides detailed explanation with suitable examples as to why having separate sets of norms for literates and illiterate groups does not satisfy the standards of intelligence testing; and attempts to provide possible solutions on whom and how this battery can be used.
\end{abstract}

Key words: Bhatia's Battery of Performance Tests of Intelligence, intelligence, IQ, performance test, battery.

(Paper received $-12^{\text {th }}$ August 2020, Peer review completed $-28^{\text {th }}$ August 2020; Accepted $-29^{\text {th }}$ August 2020)

\section{INTRODUCTION}

Bhatia's Battery of Performance Tests of Intelligence, popularly known as Bhatia's intelligence test or Bhatia's battery is one of the popular intelligence tests in the Indian subcontinent. The battery of tests consists of 5 subtests. viz Kohs' Block Design (BD), Alexander's Pass-along (PA), Pattern Drawing (PD), Picture Construction (PC) and Immediate Memory (IM) Tests. The battery is standardized on Indian boys in 1950s for ages between 11 to 16 years. The battery yields the overall Intelligence Quotient (IQ) based on all the 5 subtests and Performance Quotient (PQ) based on the 4 performance tests (BD, PA, PD and PC). The test's IQ as well as PQ range is relatively restricted and range between 69 to 131 [1]. It is probably one of the pioneer tests of IQ that dealt mainly with performance subtests/items.

Despite some of the limitations, such as that it is standardized more than half a century ago, standardized mainly on boys and that it provides IQ only between 69 and 131, many universities as well as substantial number of M.Phil. Clinical Psychology training institutes in India still teach this test. It is quite popular among many psychologists, who use it in their regular work to assess intelligence. The main reasons for its popularity are, that - 
1. It is a performance test and hence can be administered to people with language incompatibility, poor verbal abilities

2. It can be used in some cases to children suspected of Specific Learning Disability (SLD), when the examiner is sure that the child has average intelligence, but (examiner) wants to objectively prove that the child's intelligence is average

3. It takes relatively less time approximately 30 to 45 minutes

4. The subtests are interesting; where Block Design (BD) test has wooden blocks with bright colors; Picture Construction (PC) test has drawings-pictures that represents the Indian subcontinent/culture; Pass-along (PA) test have sliding parts; and Pattern Drawing (PD) test requires drawing lines with interesting conditions. This automatically increases the cooperation as well as motivation of the subjects/patients.

5. Has separate set of norms for literates and illiterates, which apparently seems appropriate, given the illiteracy rates in the country and hence appeals to substantial number of the professionals.

6. It is easy to administer and score.

In addition to the above, one of the important aspects of the test administration is that, during the administration if the subject is unable to complete the task (fails), within the time limit, on any of the levels, in the four performance subtests (BD, PA, PD, and PC); the examiner demonstrates the correct procedure of doing it, before proceeding to the next item of the test. This can actually be considered as one of the best methods to check the actual ability of the person and how well he learns and makes use of the information taught or the feedback. Rarely any tests of intelligence have this mechanism to demonstrate the correct technique/answer when one fails before proceeding to the next level.

Due to expectation on the part of the examiner to demonstrate the correct technique/answer when the subject fails, many examiners themselves find it difficult on the higher level of the test. Due to which they need to practice and master the correct technique/answer for every level of all the four subtests. It is not surprising for one to see the markings of the correct answers in the test manual of many students/trainee psychologists.

Despite the positives of the test, there are some serious issues and concerns which this article tries to discuss.

\section{ISSUES AND CONCERNS}

\section{IS THE SEPARATION OF NORMS FOR THE LITERATE AND ILLITERATE APPROPRIATE?}

One of the major things that the Bhatia's battery assumed that illiterate people as a group are different compared to literate people as a group in terms of intelligence. This assumption was based on or justified by the results obtained during the norms development. That is, the illiterate group showed significantly lower scores compared to literate group [1] (p88). This was used to justify having separate set of norms for illiterates.

The irony is that, what many professionals consider best about Bhatia's battery, that it having two-separate set of norms for literate and illiterates, in itself is the most serious limitation of the battery. The very conceptualization that literates and illiterates form separate groups, constitutes the fundamental error of assessment of the individual differences, especially the assessment of abilities, such as intelligence tests. The error of conceptualization that the illiterates and literates form separate groups, has enormous implication with respect to gross miscalculation of IQs for both literates and illiterates, thereby leading to incorrect feedbacks, erroneous calculation of percentage of disability, improper intervention or rehabilitation programs, and/or inaccurate career and future planning.

It is very surprising that till now this went unnoticed. It might so happen that even after going through this article and the explanations as well as the justifications provided, it might be difficult for few of them to accept that the Bhatia's battery lies on a shaky foundation, which can destabilize the entire assessment 
results and make the norms of the battery null and void, if certain issues are not considered. The following are some of the possible reasons as to why it might be difficult to accept that Bhatia's conceptualization of two separate set of norms as erroneous:

- The test has been in vogue since 1950s. So, the belief that, if any assessment tool that is being used from so long and not challenged till now, might be correct.

- The belief that when it is used by majority of the professionals, then it might be accepted by majority, and the associated belief that majority is always right.

- The belief that when the test is taught in many premier institutes and by eminent professionals, then it might be acceptable.

- The all known human factor that resists change, especially if it requires major adaptation and/or rejecting something which one is used to.

- Another common factor is that, the position of the person who proposes the change (identifies the error) and the position of the person/audience whom it is been addressed to. For example, if the proposing person is a young professional, then senior professionals might have issues in accepting. Similarly, age factor also works in the same way. Young professional's discoveries will not be easily accepted by the already established/senior professionals easily. The same applies to the institutional affiliation of the person, as well as where it is being published. It is easy to believe if the suggestion comes from a reputed institution as well as if it is being published in a reputed journal.

- The belief that the it is already been thought over by test developer, evaluated by test reviewers, scrutinized by the test publisher, it might be sound in its conceptualization.

- The belief that if the test has been reviewed / written foreword by a psychologist from developed countries, then it might be correct.

However, some of the professionals who resist change due to the above-mentioned reasons, rarely know/remember that many times, several good tests have been discredited/altered after few years/decades due to various reasons. For example, due to errors in the conceptualization of the test format, age-scale format was changed to point-scale format in Stanford-Binet Scales [2]. Similarly, errors in sampling and test standardization was corrected in later Wechsler's scales compared to the earlier version's sampling methods. Further, several popular subtests of Wechsler have been dropped in their latest versions and also the very way intelligence is reported has been changed from verbal \& performance IQs to Indexes [3].

Therefore, the reader is requested to ignore all the preconceived beliefs and examine the facts presented below with fresh and unbiased way.

The reasons why it is erroneous to separate illiterates and literates in the norms are as follows

\section{A. Normal Probability Curve}

It is expected and required by all the intelligence tests to adhere to the distribution of such abilities (in the general population) to closely resemble the normal probability curve (NPC). The relatively standard practice with respect to intelligence assessment is to have a mean and the standard deviation (SD) of the test to be 100 and 15 respectively [4]. Given this, test developers adjust their test items in terms of difficulty level to achieve the normative scores relatively equal to the mean of 100 and SD of 15 .

For majority of the ability tests, norms usually are developed for particular age levels. For example, separate norms for age 10, separate norms for age 12 and so on. It can be usually observed that for younger years, say till late adolescence, norms will be more distinctive. For example, 8 years, 9 years, 10 years will have separate norms. In contrast, for middle/older adults the norms cover wider age ranges. For example, the norms can be for 40 years, 50 years and/or 60 years. This is due to significant changes that occur during childhood and adolescent years, compared to adulthood.

Irrespective of particular age, the norms are usually supposed to represent all the people in that age level. So that, it can be generalized to the entire population of that particular age level. A small exception to this can sometimes be seen in earlier tests, where there were separate set of norms for males and females. 
However, in recent times majority of the intelligence tests do not have separate set of norms for males and females.

Further, if it is an intelligence test, the standardization sample is supposed to have people representing all types of IQs, such as people with intellectual disability on the lower end as well as people with very superior intelligence on the higher end of the NPC. Only then the IQs match the normal probability distribution. Hence Bhatia's battery should have also included people who are illiterate in one set of or as common/comprehensive norms. People who are illiterate, are part of population and its distribution.

\section{B. An intelligence/IQ score is a comparative value}

It is not the scope of this article to discuss what is intelligence and/or what are the components of intelligence. Irrespective of what it is, intelligence or IQ score is 'a comparative value'. That is, an IQ score of a person is derived in comparison to people who belong to her/his age group. This is popularly referred to as 'deviation IQ' (Even in age scale-ratio IQs the age comparison would have done during the placement of items for particular age groups). That is, for example, if a person gets an IQ of 115 (i.e. mean $+1 \mathrm{SD}$; according to the WHO recommended norms), then approximately, about $84 \%$ of the people have less intelligence (or $15 \%$ have more intelligence) than him/her. Therefore, an IQ score of a person is always in comparison with her/his age group. Given this, as mentioned above in section 1.A. a normative sample should have all types of the people in that particular age group, including people who are illiterates.

\section{C. Illiterates are also part of the same population}

Closely related to what is mentioned in section 1.B., illiterates are part of the population (society/country) and they should be considered a part of the same norms. While developing norms one will not have separate norms for low vs. high socioeconomic status groups; high vs. low education groups; and/or different norms for different castes, religion or ethnicity. This is because, all these people belong to the society/population of which we derive the norms and for which the generalization will be made.

There exist innumerable groups in a society. It can be caste, religion, race, language, dialects, geographical region, socioeconomic status, occupation, gender, age, education, and so on. An individual usually belongs to several groups at the same time. That is, a person can be 16 years old girl studied upto 10th grade, from rural areas of Bengaluru belonging to middle socioeconomic status of xyz caste in xyz religion of xyz race who speaks xyz language.

Given this, a test that assesses intellectual ability cannot have multiple groups (except developmental age), such as separate norms for different castes, different religions, different regions, different socioeconomic status and so on. For example, Bhatia's norms showed that as a group, children of parents belonging to 'higher professions' (that included lawyers, doctors, engineers, teachers and high government officials), had an average IQ of 106, compared to children of parents who are involved in 'agriculture' (including landlords) had an average IQ of 91 [1]. So here, just because children of higher professions showed significantly higher IQs compared to children of farmers, it does not mean that they both should have separate set of norms. Similarly, just because people who are illiterate showed less intelligence as a group (compared to people who are literate), it should not mean that there is a need for separate set of norms for people who are illiterate.

\section{D. Probable effect of schooling on the group differences.}

There is no unequivocal agreement on why and how intelligence varies, if at all, with respect to different racial, cultural and environmental conditions. Further, it is also commonly known that, there are no intelligence tests that can provide justice to all the various groups or subgroups. It is often said that, culture- free test is an illusion, because irrespective of whether the testing material (i.e. verbal-nonverbal, mathematical, artistry or behavioral), culture has significant influence on intelligence and/or how it is tested.

Intelligence tests only assess the levels of intelligence in a group. It is based on, and limited by the sample selection as well as the items that are used in the test. Intelligence tests do not and cannot identify the cause of differences in intelligence among different groups, be it racial, caste, and/or geographical region. 
In this regard, it is rightly said that ".... intelligence tests do not in themselves enable us to differentiate safely between what is due to innate capacity and what is the result of environmental influences, training and education. Wherever, it has been possible to make allowances for differences in environmental opportunities, the tests have shown essential similarity in mental characters in all human groups" [5].

However, the significant difference in the performance between the literates and illiterates can be relatively attributed to schooling to some extent. Bhatia also attributes the difference observed between the groups to "formal school practice and the demands of civilization" (Bhatia, 1955). This is because, both the illiterate and literate groups had children from rural areas, and even though not equally, both parents belonged to relatively similar occupation, caste/religion and socioeconomic status (for further discussion in this regard, please refer sections 1.E. to 1.I). The only major difference that seem to be apparent is that one group was in school and another group was not in school and/or did not know how to read and write. Therefore, the only probable reason that the difference in intelligence can be attributed to is schooling.

However, one can argue that, the battery contains performance subtests, which are primarily meant not to be adversely affected, due to lack of formal education. In addition, manual clearly mentions that the examiners took great efforts in making themselves familiar to, and establishing good rapport with the community as well as the test taker. It also mentions that children were given adequate practice before starting the actual assessment. So, to some extent all the children would have had familiarity with the test materials and good rapport with the examiner. Therefore, to continue this argument, it can be debated that the performance tests should have reduced the difference in the IQs.

On the other hand, it can also be argued that, the observed differences (which is quite high) in IQs between the groups are actually less, and if non-performance tests were used, the observed group differences would have been even greater.

Despite the above argument (that it is performance battery and rapport was established), it is beyond doubt that the education does play a role in determining one's level of intelligence. It is common knowledge that IQ and academic achievement are highly correlated, and in general higher IQ yields better academic grades. On the other hand, school education also contributes to IQ, where a large meta-analysis found that an additional year of schooling can contribute to about 1 to 5 points increase in IQ and has long-lasting effects [6].

However, few things that does matter are

- One cannot create separate norms just because a major chunk of the population did not go to school. If still one goes ahead and creates separate norms for them then, it will be akin to creating separate norms for a particular caste or low socioeconomic status group (a major chunk in India)

- A battery of tests that has performance subtests, with culture \& illiterate friendly material does reduces the negative effect of not having gone to school, with regard to intelligence assessment (refer section 1.G. for detailed discussion in this regard). But, the extent of such reduction is very difficult to estimate.

- If school attendance alone was the factor that determined the differences, then, the significantly lower results observed in the immediate memory for illiterate group could not be present to the extent that was observed, as the test involved repeating verbal sounds that people use in their daily life (refer section 1.H. for a detailed discussion in this regard).

- It can be argued that, as mentioned above, the observed group differences (which is larger) in Immediate memory subtest was actually lesser, and if numbers of regional language were used instead of regional- verbal sounds, the group difference would have been even greater. However, though this argument seems plausible, it cannot be fully considered as true. Because, the group differences observed in Immediate memory is almost similar to that of other subtests. That is, if the regionalverbal sounds did reduce the group differences, then the group differences in Immediate Memory subtest would have been lesser compared to other 4 subtests. However, on the contrary, it is surprising to observe that despite using regional-verbal sounds (instead of numbers, which were difficult), illiterate group performed significantly worse (in comparison with literate group) in the Immediate Memory subtest compared to other 4 subtests (refer the bar diagram of IM, in figure 1). 
Therefore, the differences observed between groups cannot be solely and fully attributable to differences in schooling.

\section{E. Illiterate vs. Intellectually inferior}

One of the main short-coming of the battery is, not considering the reasons for children being illiterate. Compared to recent times where prime importance is given to school education, in 1940-50s India not many parents sent their children to school, probably due to lack of awareness, lack of opportunities (such as school availability, long distance, lack of toilet in the school) and/or the poor socioeconomic conditions.

However, one major reason could be that the children (at least some substantial number) in the illiterate group might have been really 'intellectually inferior', and hence they might have scored lower IQ. That is, as a group, illiterates scored less IQ, probably because the group contained more children with intellectual disability and not because they were illiterate per se.

Apart from the extraneous factors (eg. lack of opportunities, poverty, socioeconomic-political reasons) that might be responsible for a child not going to school, there can be intrinsic factors within a child that might have contributed for him not going to school. These factors can be 'global developmental delay / intellectual disabilities'. It is a common knowledge that if the child has any global developmental delay or intellectual disabilities, parents will not (or find it difficult to) send the child to school and/or probably school might not admit (or let it continue to attend) the intellectually disabled child. On the other hand, it is also common that children with mild intellectual disability might find it difficult to cope-up with the academic demands, and can drop out of the school during primary classes (before they reached 11 years, which is the starting age of this battery).

Given this, if this child is taken for the standardization and put into the illiterate group, it is natural that this child will perform poorly on an IQ test compared to a child who is literate. Therefore, this poor performance/IQ cannot be attributed to the child being illiterate. One important justification for the above argument is the performance of the illiterate group in Immediate memory subtest (refer figure 1 and section 1.H for detailed discussion).

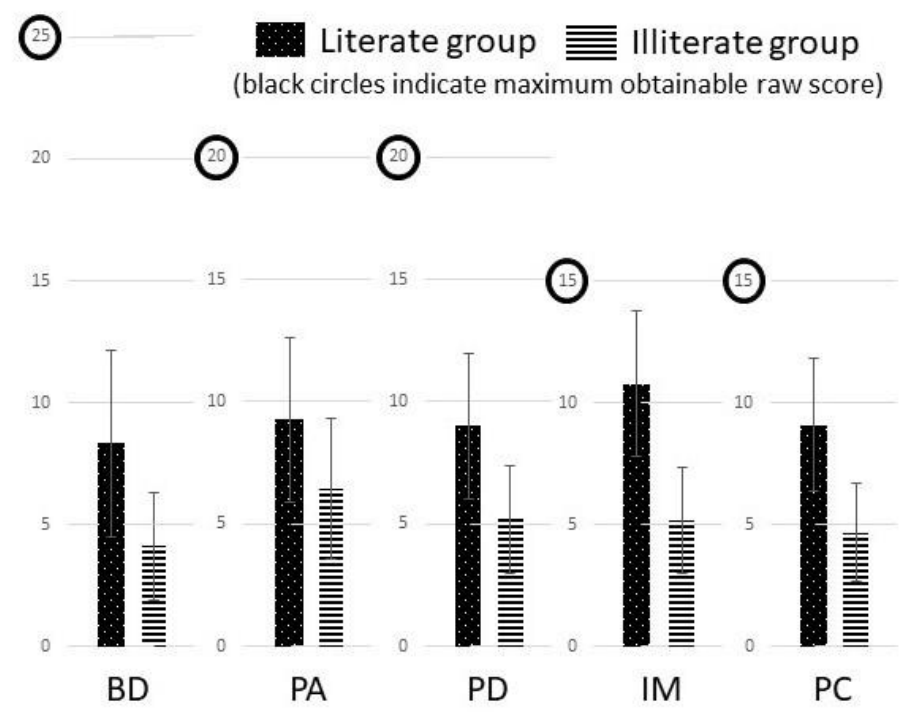

Figure 1: Showing the raw scores of Literate and Illiterate groups across different subtests

In addition, the selection of the sample also supports the above to a great extent. The sampling for the two groups in the standardization were completely different in terms of caste/religion (refer table 1) and parents' occupation (refer table 2). For example, the illiterates group included about $63 \%$ of children from 
the so- called "backward communities" or "Harijans", which currently referred to as 'scheduled castes and scheduled tribes' (SC and ST respectively). In contrast, the literate group included only about $5 \%$ of children from the so-called "backward communities" [1].

Table 1: Showing the differences of the two groups in terms of the distribution of the caste-religion of the normative sample.

\begin{tabular}{|c|c|c|c|}
\hline & Categories & Illiterate group & Literate group \\
\hline & $\begin{array}{c}\text { Non-backward community } \\
\text { (Brahmin, Kshatriya, Vaishya, Muslim) }\end{array}$ & $36.58 \%$ & $85.66 \%$ \\
\cline { 2 - 4 } $\begin{array}{c}\text { Caste/group } \\
\text { and Religion }\end{array}$ & Backward / Harijan community (i.e. SC and/or ST) & $63.42 \%$ & $4.83 \%$ \\
\cline { 2 - 4 } & Others (eg. Jains, Parsis, Sikhs) & No information & $9.5 \%$ \\
\hline
\end{tabular}

Similarly, in terms of occupation, illiterate group had about $62 \%$ of the children whose parents were Farmers, compared to only about $14 \%$ in literate group.

Table 2: Showing the differences of the two groups in terms of the distribution of the occupation of the parents in the normative sample.

\begin{tabular}{|c|c|c|c|}
\hline \multicolumn{2}{|c|}{ Illiterate group } & \multicolumn{2}{c|}{ Literate group } \\
\hline Farming / agriculture & $62.50 \%$ & "Higher professions" & $21.03 \%$ \\
\hline "Small village Shopkeepers" & $7.81 \%$ & "Middle class service" & $35.36 \%$ \\
\hline Artists & $11.33 \%$ & "Lower class service" & $7.32 \%$ \\
\hline Hired laborers & $5.66 \%$ & Business & $12.93 \%$ \\
\hline Domestic helps & $8.40 \%$ & Agriculture & $14.33 \%$ \\
\hline Not known / unemployed & $4.29 \%$ & Not known & $9.03 \%$ \\
\hline
\end{tabular}

This article is limited in its scope and does not discuss the merits or demerits of belonging to particular group in terms of caste, religion, and/or occupation. However, given the sociocultural milieu of 1940-50s during which the standardization of the battery took place, one cannot ignore the differences (in terms of the sociocultural-economic conditions), and how such differences could have contributed to the variations in terms of learning potentials and abilities.

These differences between the two groups in the representation of children from varied caste, occupation and socioeconomic conditions should have been controlled or reduced in the standardization sample. If the sampling were more equally distributed and/or more fairly representative, probably the differences observed between the groups would have been less, and subsequently there would have been no reason to have two set of norms.

To reiterate, even though there exist differences in the IQs between literate and illiterate group and that difference might have been the result of sociocultural-economic conditions, one cannot have a separate set of norms for literate and illiterate groups.

\section{F. Plateau in performance}

Literate group showed relative plateau in their performance (on the higher side/scores) compared to illiterate group. The illiterate group did not show this plateau in any of the subtests except Picture construction (fig. $2-6$, pages $83-87$ [1]). Figure 2 depicted below is an attempt to explain the above phenomenon. Due to the possible copyright issues, figure 2 has been modeled on the results observed in the standardization sample, and not the exact replication.

Just for discussion purpose here, if we consider that, if in case, the examiner takes the illiterate group norms for an illiterate person who is 16 years old, and certifies a particular intelligence based on how the person is performed in the test. Given the standard belief that IQs do not increase beyond 16 years, the examiner 
who wrote the report, the person who reads the report, as well as the subject, all believe that the IQ mentioned in the report is the exact/true representation of his abilities. However, as the norms itself has suggested that there is no plateau/ceiling observed in the performance in illiterates, it suggests that their performance might improve over and above 16 years. Given this, it would be unfair for any person who is an illiterate if one uses the separate set of norms meant for illiterates.

Hence, the above fact again emphasizes that the separate set of norms for illiterates is not an appropriate idea.
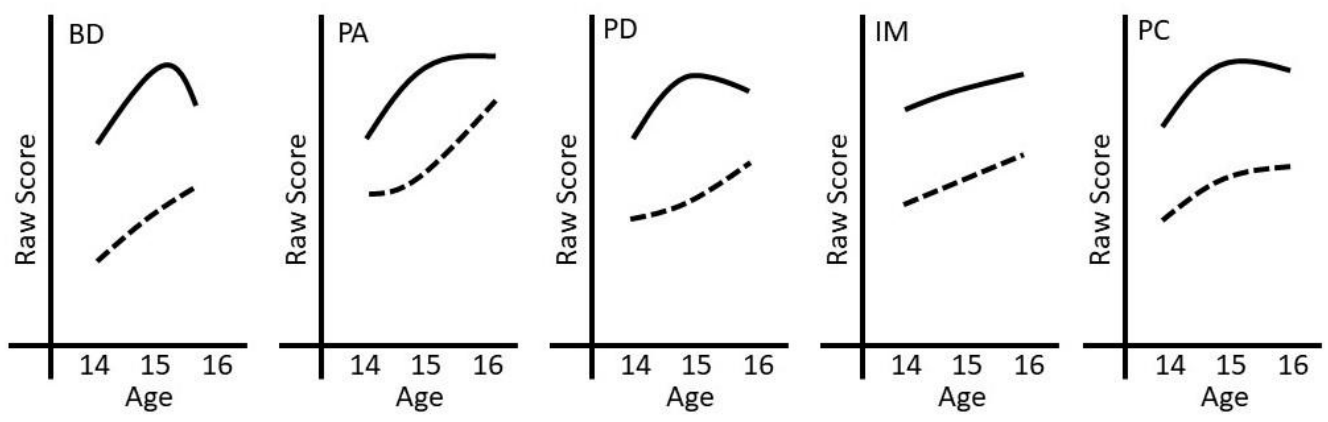

— Literate group ---- Illiterategroup

Figure 2: Depicting Performance plateau in literate group

\section{G. Performance tests}

It is usually accepted that nonverbal performance tests are the best option to administer if the subject does not know the language of the test and/or if the subject has poor verbal skills. Indeed, this is the case in India, where, many psychologists resort to Bhatia's battery and/or Standard Progressive Matrices.

As mentioned in section 1.D., some form of formal education always benefits in one getting a good score even on nonverbal or performance tests. However, the lack of the same (school education) does not in any way bring down the IQ substantially, as observed in the standardization sample.

One of the justifications put forward to develop this battery was that, "...tests of intelligence which do not require reading and writing on the part of the subject". Further, it is mentioned in the battery manual that, '... life in the village, again, is practically all out of doors. The village boy spends his day out in the fields, helping the elder folk in cultivating or in tending cattle. The experience that he comes across is the handling of concrete objects and materials".

Given this, it can be assumed that all the people, especially the illiterate group where majority were from the rural area, might not have been so unfamiliar with the performance subtests used in the current battery. This is because, the battery consisted of colorful blocks, moving blocks, arranging pictures, drawing lines and repeating numbers/syllables.

Picture construction test consisted of pictures with human and livestock that depicted rural environment. According to the battery manual, pattern drawing was actually developed based on the observation of the common pastime in rural settings. Immediate memory had material familiar to the literate as well as illiterate group.

The subtests that were probably unfamiliar were the Kohs' block design and Alexander Pass-along test. However, this unfamiliarity might have been similar to both literate as well as illiterate group. Children from both the groups would have played and/or had familiarity with indoor \& outdoor games played by people irrespective of whether they are from rural-urban and/or literate-illiterate background. Examples of such indoor games are, lagori, kancha/marbles, gilli danda, top/lattoo, snakes $\&$ ladders, cross $\&$ circle game (Pachisi), dice games (chauka bara / Dayakattai), and games played with small stones/conch shells. 
Given this, it cannot be said that being illiterate would have put the children in such a disadvantage as the results obtained in the normative study of a performance test of intelligence. Hence, it would have been better to have a single comprehensive set of norms, instead of separate sets of norms.

\section{H. Immediate memory}

It is very interesting that to overcome the hurdle of illiteracy, the Immediate Memory subtest adopted verbal/speech sounds instead of numbers. The test did not even use the regional language numbers (eg. Ek, Do, Teen). Given this, it is very surprising that, despite having accommodated for the shortcoming of illiteracy, the manual created a separate norm for illiterate group.

An analogy can explain this better. For example, let us assume that out of two long distance runners, one runner needs to be selected to represent Karnataka state for a national level competition. One of the runners, 'Rura' is from rural background and the other runner 'Urba' is from urban background. A coach decides to have a race to select the best runner. The coach realizes that 'Rura' does not have shoes to run. Due to it, the coach decides that it would be unfair to make him run without shoes. So, the coach gives a pair of roller- skates to 'Rura'. Race begins, where 'Urba' runs and 'Rura' skates to covers a distance of 20 kilometers. 'Urba' reaches first and 'Rura' despite using roller-skates reaches second. However, the coach decides that it is still unfair to 'Rura', as he might not be familiar with roller-skates. Therefore, coach does not recommend either of them to the running race.

In this analogy, the issue is not about the fairness, equality and/or justice. It is about selecting a person to run the race to represent one's state/region. The issue was not about one's region, it is about running and the race. Given the same, intelligence assessment is about assessing where does the person stand among his age peers. It is not the question of 'whether one should do the assessment or not', 'whether who gets more or who gets less IQ', and/or 'what is the reason for one getting the lower IQ'. If one decides to do the intelligence assessment, then the result will always be comparative (as mentioned in section 1.A. and 1.B above).

Another interesting fact is the aspect that verbal-speech sounds (used in this battery for illiterate group) is not an exact substitution for numbers in the assessment of immediate memory. Actually, for three main reasons the verbal-speech sounds are far too easier to recall than the numbers.

First, each verbal-speech sound used in the battery (to assess immediate memory of illiterate group) constitutes just one element of sound, almost similar to that of phoneme. For example, $\mid$ sa $|$,$| la |,| \mathrm{ba} \mid$, $|\mathrm{ma}|,|\mathrm{ta}|$. Compared to this, the numbers used in the battery (to assess literate group) constitutes more than one sound or more than one phoneme. For example, 'four' has about 2 sounds, i.e. $\mid$ fo $|+| r \mid$, and 'seven' has about 3 sounds, i.e. $|\mathrm{Se}|+|\mathrm{Ve}|+|\mathrm{N}|$.

Given that, the capacity of the attention/working memory span is about $7 \pm 2$ bits of information, it is easier to register and recall more of verbal-speech sounds compared to numbers. For example, ' $|\mathrm{sa}|,|\mathrm{ba}|$, $|\mathrm{ka}|,|\mathrm{da}|$ ' is just 4 bits of information, and whereas 'eight, three, seven, five' is about $(3+2+3+2) 10$ bits of information. Research supports the above assertion. For example, it has found that Chinese children has better forward digit span (recall more numbers) compared to language such as English. This has been attributed to the fact that many Chinese numbers are pronounced with mainly one sound, for example, $\mid$ yi $|$,$| si |$,$| qi| [7-9].$

Second, it is easy to register and remember the verbal-speech sounds compared to the numbers, due to the simple fact that the verbal-speech sounds can be automatically combined to make words. For example, one of the tasks that requires the subject to recall/repeat the vernacular verbal-speech sounds are 'pa, cha, la, ra, ba, sa' (which is a substitution for 6 digits forward recall). Here it is clear that the subject can easily make up words that are easier to remember, because it is in words format (chunks/group), the chunk might sound similar to real words. For example, the above speech-sounds can be chunked as 'pachal rabas'.

Third, the familiarity with the verbal-speech sounds used in this subtest far exceeds the familiarity of the numbers. That is people's entire conversation in their day-to-day life involves the very speech sounds used in the subtest to assess immediate memory. Therefore, automatically the test stimulus will be very familiar 
to the illiterate group. In comparison, even though people in the literate group use numbers in their daytoday life, it is far less familiar compared to speech sounds.

Given the above-mentioned advantages, it is clear that recalling verbal sounds is far too easier than recalling numbers even for illiterate group. However, it is very surprising that the illiterate group performed significantly poor $($ mean $=6.95 ; \mathrm{SD}=2.04)$ compared to literate group (mean $=10.77 ; \mathrm{SD}=$ 1.84 ) in immediate memory subtest, which is about $35 \%$ lesser (refer figure 2 ). The possible reason one can attribute closest is to the factors mentioned in 1.E - Illiterate vs intellectual inferiority.

\section{I. What purpose does the separate norms for people who are illiterate serve?}

As mentioned above, having separate norms for illiterate group appeals to some professionals/trainees. It might appear that it is 'just and fair' that a section of people should not be penalized for no fault on their part that they could not go to school. This belief usually arises from the fact that if illiterate person is compared with common norms that involve both illiterate and literate people, then the illiterate person will get lower IQ. Here, this issue seems to loosely resemble the dilemma of social justice or social efficiency, and it appears fair to support social justice. Given these beliefs, some might think that having separate norms will benefit and it will do justice to the illiterate group.

However, rarely one thinks about what and how are these benefits help the illiterate group, if there is a separate set of norms for them. Apparently, there seem to be no actual benefits for the people who are illiterates for having separate set of norms, be it in relation to school, job, availing benefit schemes/services of the government, legal issues and/or rehabilitation. On the other hand, having separate set of norms can adversely affect the people who are illiterate. Some of the examples below will clarify how it might actually not help the people who are illiterate.

\section{I.i. School:}

Usually there are two main reasons why schools refer children for the assessment of intelligence, one for admission purpose, and the second when the child is showing poor performance in academics. This is done to see whether the child has any developmental delays.

This battery cannot be used for both of the above purposes because of two main reasons. One, the battery is standardized for children only between 11 to 16 years. In addition, rarely any child after 11 years tries to join a school. Second, for the obvious reasons that there is a separate set of norms for illiterate people. If this separate set of norms is used then a child might be considered as average, but might not perform better in school at all (refer section 1.J. for a detailed discussion). An example might make it clear. Let us assume that there are no separate norms for illiterate and literates, i.e. there is only one set of norms (that includes all types of children, literates and illiterates alike, based on the general distribution of the population). Given this, if a child still wants to get admitted to the school, if in case, the norms would have told where the child stands with respect to others in the class (for more clarity refer section 1.J. on Disability assessment). Given the above, a separate set of norms for the illiterate group was not warranted.

\section{I.ii. Job}

There is hardly any employment recruitment agency anywhere in India that expects IQ score to recruit (for a job) a person who is illiterate. Similarly, there won't be any demand for an IQ score for any job promotion. Recruitment for any training and/or job that requires reading and writing usually depends on the academic level/degree a person has attained. For those jobs that do not require reading and writing, none will require the person to furnish IQ score. Therefore, with respect to any job recruitment per se, there was no need for a separate set of norms for illiterates.

\section{I.iii. Clinical purpose}

It is a common practice in several mental health institutions and hospitals that patients are referred for intelligence assessment for various purposes. Apart from the purpose of disability benefits, IQ assessment is carried out to decide the plan intervention/treatment, to decide whether the person can benefit from the psychotherapy process, to determine the prognosis, to provide psychoeducation and for the rehabilitation purpose. However, using a separate set of norms for illiterates actually work against the patient and their family. 
What a separate set of norms for illiterates does is, it actually automatically and artificially elevates just the IQ score without elevating the actual ability of the person who is illiterate. That is, the obtained IQ after using the separate norms (for illiterates) will be higher than what actual IQ. With this incorrectly elevated IQ, any plan for intervention, psychotherapy, psychoeducation and rehabilitation is bound to have some limitation. For example, if the person with actual 'borderline' level of intelligence is administered this battery and referred to the norms for illiterates, then the result might indicate that he has 'above average intelligence'. Given this, (incorrect, but not known) the examiner can say that the person can do well in therapy, job and life. However, it might not happen because actually the person has 'borderline' level of intelligence. For detailed discussion in this regard, refer to section 1.J. - Disability assessment and particularly the figure 3 ).

\section{J. Disability, Legal issues and rehabilitation}

Generally, one can have a misconception that the separate set of norms will benefit the illiterate people in availing any government schemes, legal issues and rehabilitation. However, it is a misconception, and in contrast, a separate set of norms actually discriminates against the illiterate person in the society.
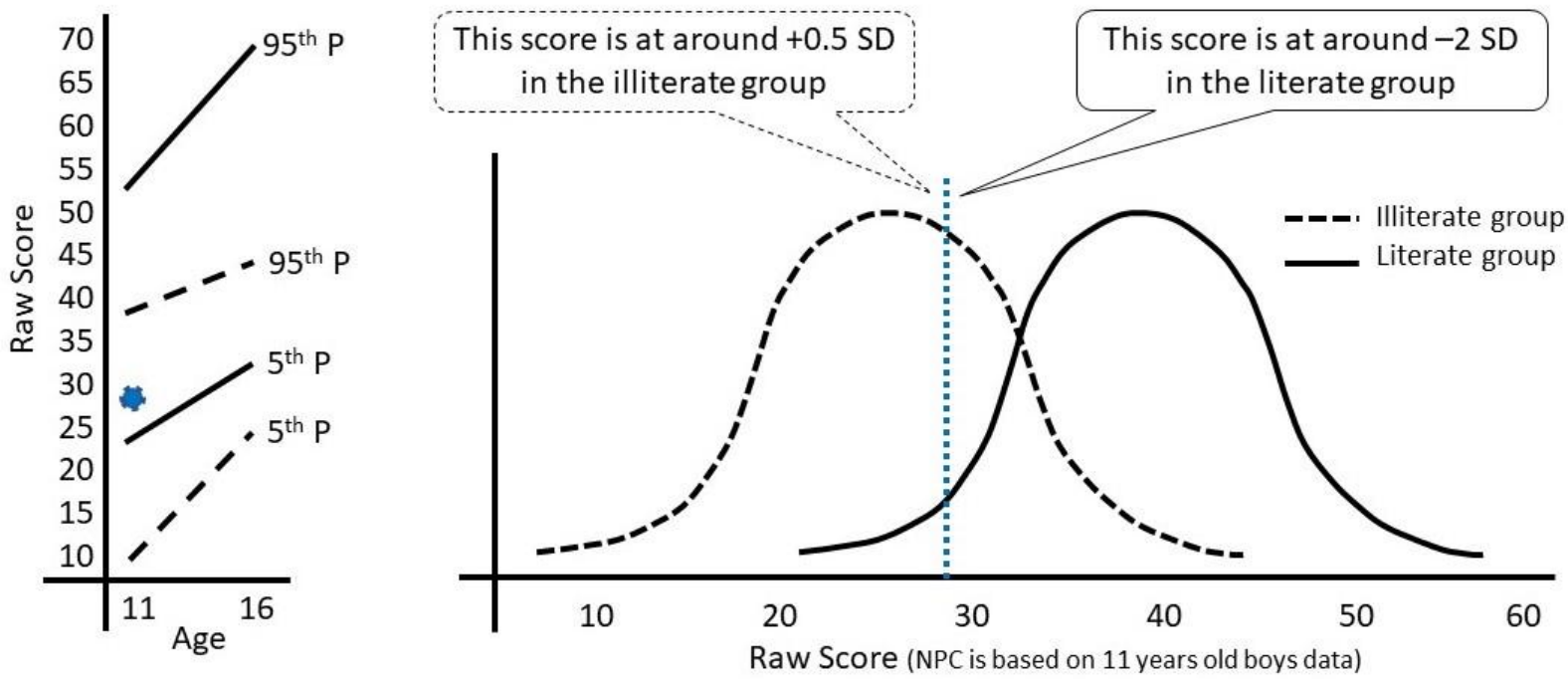

Figure 3: Percentile obtained for illiterate and literate group

The blue small circle seen in the left figure corresponds to the blue small-dotted line on the right figure The above two figures are drawn based on the data depicted in the manual (Bhatia, 1955)

As it can be seen in figure 3 that two sets of norms will have two distributions. The two curves drawn on the right of the figure 3 are based on the 5th and the 95th percentile ranks of both illiterate and literate groups of 11 years old boys, as depicted on the left side of figure 3 (as well as in page 88 of the manual; [1]). For explaining purposes, the distributions are drawn resembling normal probability curve. The blue vertical dotted line in figure 3 shows how a particular raw-score can have a different interpretation based on different distribution/norms. Let us assume that the raw-score (the blue vertical dotted line) is 28 . Then this score might correspond relatively to above average intellectual ability if illiterate norms are used. On the other hand, the same score might correspond relatively to 'borderline' intelligence category if literate norms are used.

Given the above, let us assume that the parents bring their son, of 11 years old, not gone to school boy (eg. Master Bor), to a psychologist. They say that the Government Census guy (who visited them from the city) told them to contact the psychologist to claim disability benefit for Master Bor, because the Census guy has felt that the child has intellectual disability. However, parents say that they don't think he has such a problem, because the child takes care of self-help needs, feeds cows, cleans the cowshed and goes around 
their small village on his own. However, they report that the child had small delay in speaking (which they further say is commonly seen in their family), dropped out of school at 1st grade (again they report that it is a commonly seen phenomenon in the family and neighborhood), and due to which he does not know calculations, and hence they don't send him for shopping with money, and so on.

The psychologist administers the battery and the child gets a raw score of 23 . The psychologist uses the norms meant for illiterates and determines that the child's IQ is 103 (refer left-side NPC on right part of the figure 3 above; as well as the manual norms on page 123, Bhatia, 1955). Therefore, the psychologist says that the child has average intelligence and says that the child does not have any intellectual disability. This might seem fair, as the psychologist has used the norms meant for illiterates. But technically it is not correct and with respect to disability assessment it is a serious mistake. This is because if you look at the green color NPC (which would have been the actual one comprehensive norms; read section 1.K. for clarity on this) in figure 4, the raw score of 23 might be somewhere in 'borderline' category of intelligence and probably the child would have got at least $25 \%$ disability.

To understand why it is a mistake in terms of disability assessment, one has to know the actual meaning of disability.

A disability can be defined as any condition in a person that makes it difficult for that person to perform certain activities and it limits his/her interaction with the world, which in turn affects her/his functioning, and thereby adversely affect the livelihood and wellbeing of the person [10].

It should be remembered that disability (diagnosis / assessment / certification) is usually independent of the cause of such disability. That is, disability might have been due to congenital, biological, developmental, acquired illness/conditions and/or accidents (or a combination of these). Again, to reiterate, it does not matter how the disability has happened, and what matters is the 'disability' itself.

One aspect of the disability is, that it is always in comparison to a general population. That is, for example, a person's seeing/hearing ability is compared to the general population to determine the disability. That is if a normal person vision is $20 / 20$, then if a person has $20 / 40$ (depending on the country/criteria) he is considered as having $20 \%$ disability. A person with disability is never compared with a group who has similar disability, to calculate the percentage of disability. That is, for example, let us assume that Mr.Onle has one leg. Then we cannot determine his disability by conducting a running/hopping race among several people who have only one leg. Further let us assume, an organization conducts such race and about 12 people with one leg participate. Everybody runs/hops the distance of say 100 meters and complete it between 120 to 150 seconds. Mr.Onle takes about 125 seconds, which is relatively faster when compared among the 12 people. Given this, one cannot claim that as Mr.Onle is one of the fastest, therefore he doesn't have disability, and disability benefit cannot be given to him.

Similar to the example mentioned above, just because there is a separate set of norms for illiterates, we cannot use it to assess it for disability.

For disability assessment IQs should go as low as 'less than 20' (in terms of WHO IQ classification criteria, 1992), so that, it can indicate what level of mental retardation/intellectual disability and what percentage of disability a person has.

The Bhatia's battery does not fully assess for disability per se, as it does not provide IQs below 69 . However, a psychologist can get adversely influenced by the scores obtained by this test (as mentioned above with the example of Master Bor) and s/he might think that the child has normal (or below average) intelligence and does not have any intellectual disability. Given such result and subsequent erroneous in interpretation, a psychologist might not even attempt other tests to see whether the child has disability or not.

The same explanation applies to any legal issues. There can be several legal related scenarios where accurate representation of a person's intellectual ability is warranted. These reasons, can vary from the capacity to stand trial, taking care of one's property, parental responsibility, guardianship purposes and so on. As explained in the above example of Master Bor, another illiterate child who actually has mild mental retardation/intellectual disability, might get a result of below average intelligence if the separate norms for illiterates is used. 


\section{K. Limitations of the norms for 'literates'}

The above discussions are mainly meant for explaining the limitations of using the norms meant for illiterate. However, one should remember that the norms meant for 'literates' is also not a proper representation of the entire community. That is, it is not comprehensive enough that can be applied to all the population. This is because, it has omitted a significant chunk of the population (i.e. illiterates), from its standardization. It is like removing the poor performers (the norms show that illiterates performed significantly poorly compared to literates; Bhatia, 1955) from the standardization. This has resulted in pushing the normative curve of the literate group towards higher side or towards the right.

Figure 4 clearly explains this phenomenon. In the figure it can be seen that a raw score of about 28 is around 'minus 2SD' if literate norms is used, which is equal to that of an IQ of about 70 (refer figure 5 for the distribution of IQ scores in NPC based on WHO guidelines). However, as this separate set of literate norms did not have an adequate representative of the population, the IQ of 70 is incorrect, and the IQ should have been little higher. If in case, the population was adequately represented, then there would have been one set of norms that included both literates and illiterates. This combined/comprehensive set of norms would have been at the green color NPC shown in figure 4. Therefore, if this comprehensive set of norms (green color NPC in figure 4) is used the person obtaining a raw score of 28 would be around 'minus 1SD', which is equal to that of an IQ of about 85 (refer figure 5 for the distribution of IQ scores in NPC based on WHO guidelines).

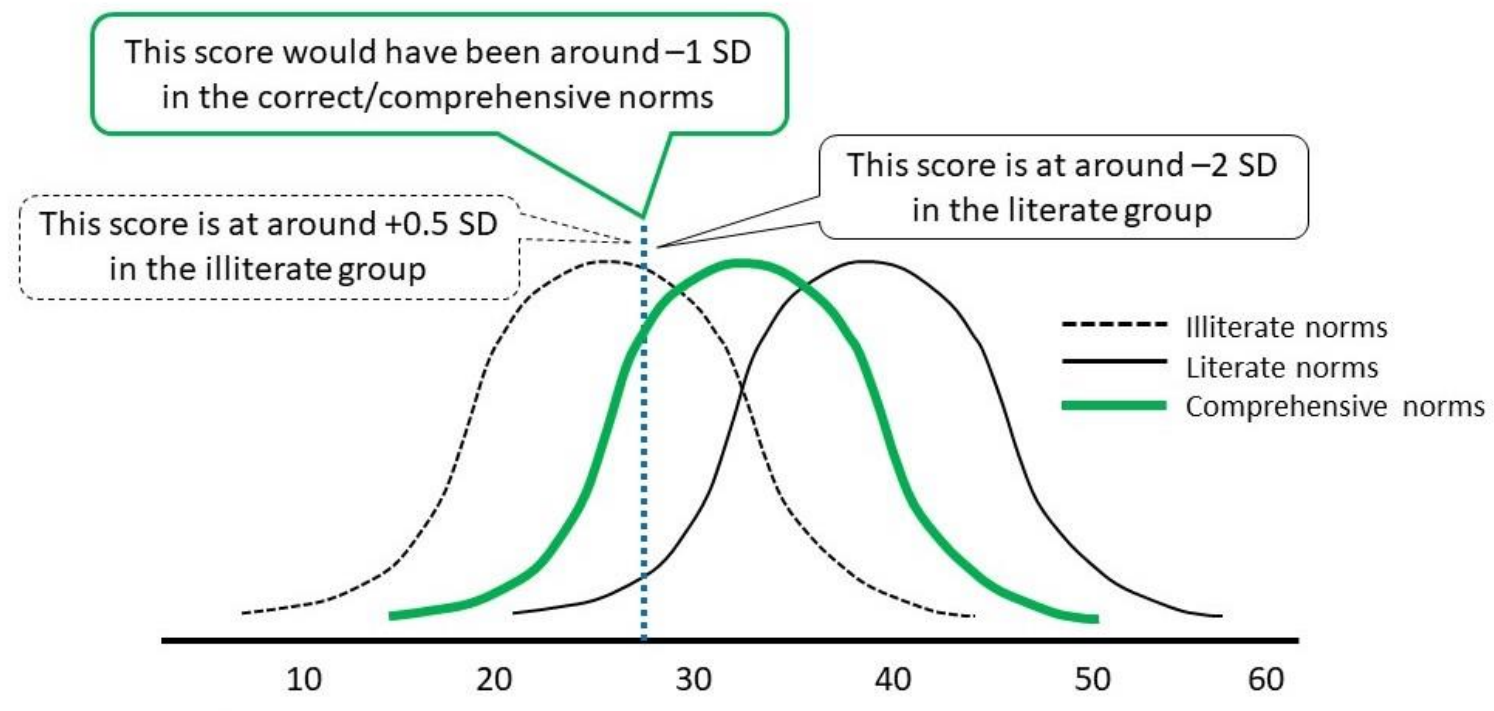

Raw Score (NPC of literates and illiterates are based on 11 years old boys data)

Figure 4: Percentile obtained for illiterate and literate group \& hypothetical correct norms The two NPCs of literates and illiterates are drawn based on the data depicted in the manual (Bhatia, 1955)

An analogy will help to clarify the above clearly.

Scenario/Time 1: Let us assume that a teacher, Ms. Bhavya wants to test where Master Dulav stands in his class in terms of running. The teacher decides to conduct a race for the class that has 40 children. On the race day, she notices that five children are having cold, 10 children appear weak and 5 who are not interested to run. Therefore, she decides to conduct the race for the remaining 20 people. Master Dulav comes in 16th place out of 20 children who ran the race. This can be said to be somewhat equal to about 25 th percentile (due to 16 out of 20 being in the lower fourth quarter). Given this, Ms. Bhavya decides/certifies that, Master Dulav is a poor runner in his class.

Scenario/Time 2: However, couple of weeks later, another teacher Ms. Roopa, without knowing the above results, conducts the race again. On the race day 5 children say they don't want to run, and 10 
children appear weak. However, Ms. Roopa encourages everybody to run as much as possible. This time Master Dulav comes in 18th place out of 40 children. This is somewhat equal to about 45 to 50th percentile (due to 18 out of 40 being almost in the mid-point). Given this, Ms. Roopa decides/certifies that, Master Dulav is an average runner in the class.

Applying the above analogy to the battery and its norms, Scenario/Time- 1 is what the separate set of norms for literates does to a child. As the literate norms does not have representatives from all types of the population (i.e. in this case, omitted all the illiterate children), the IQ score obtained will actually be lesser than what the child's potential / standing in the community. Therefore, to obtain a correct estimate of one's intelligence, the scores need to be compared with all sections of the society, as described in scenario/time- 2 (also refer to sections 1.A to 1.C above). This does not mean that one should merge the above two sets of norms to obtain one set of norms (refer 1.L.iii. for the detailed discussion in this regard).

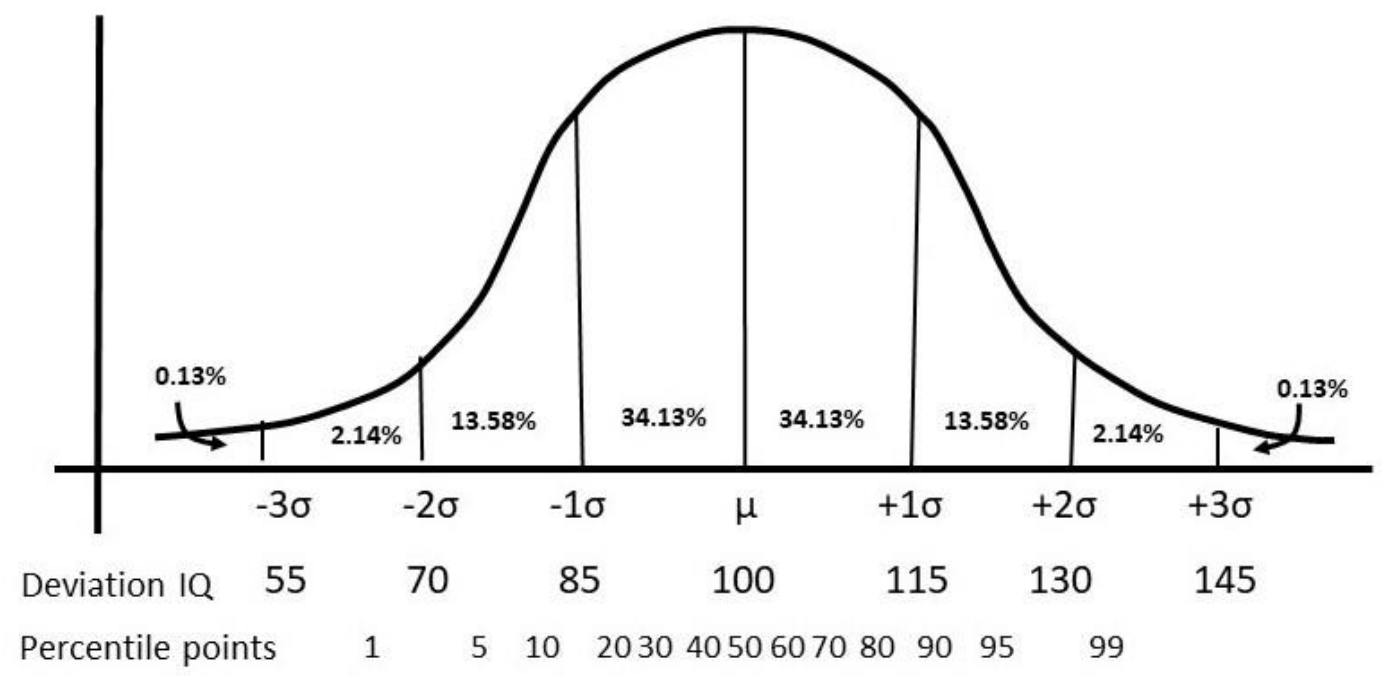

Figure 5: NPC characteristics and the IQ distribution based on WHO guidelines

\section{L. Is there a way out from the two-separate set of norms?}

Given the above limitations of having two separate set of norms, one can ask what is the solution. Does completely omitting the norms for illiterates and using only norms meant for literate help? Does one should not do intellectual assessment for illiterates? Is it possible and desirable to merge both the norms? There are no correct foolproof solutions for the above questions. The following solutions are the opinion of the current author. However, one is expected to make up their own informed decision based on the facts/ideas presented in the article or other sources.

\section{L.i. Does completely omitting the norms for illiterates and using only norms meant for literates} help?

The above scenario/time-2 (Section 1.L.) is just an analogy, and it would be difficult to exactly calculate/identify how much the NPC/IQ is actually got shifted upwards/right side for the literate group. However, this alone cannot be used to completely discredit the battery. There is hardly any intelligence test that does complete justice to each and every person/child in any given society/community. Popular and well standardized intelligence tests such as Stanford-Binet and Wechsler's tests too had significant limitations earlier in their standardization, such as, not being representative of their entire population and/or not sensitive enough to certain groups/races/communities.

As mentioned in above sections, using separate set of norms for illiterates apparently does not serve any purpose, and in turn, it affects the illiterate population adversely, in terms of disability benefit, 
rehabilitation, and so on. Therefore, as the answer the above question is 'yes'. Using only literate norms for those who are literate can be carried out with appropriate caution (as mentioned in section 1.K.).

\section{L.ii. Does one should not do intellectual assessment for illiterates?}

A person illiterate or literate has all the right to get tested for intelligence if one wishes to do so. However, there should be appropriate norms that takes into account the important factors of assessment of abilities in a given population. Given the above, one cannot say that intellectual assessment for illiterate people should not be done. However, if Bhatia's battery with norms for illiterate group is used, it will adversely affect the illiterate person. Therefore, it is strongly advised not to use illiterate norms and/or literate norms for illiterate people.

However, illiterates should be tested using a comprehensive well standardized norms that included subjects from all sections of the society. As it is not done with Bhatia's battery, it is good to not use this battery for illiterate people on the whole.

\section{L.iii. Is it possible and desirable to merge both the norms?}

It would not be easy to attempt such a task. If one tries hard, they can come up with one set of merged comprehensive norms. However, the question is, will it be correct? Few things one has to remember that, the situation and the population distribution in 1940-50s was different in terms of literacy. That time the illiteracy rates were significantly higher compared to current times. Therefore, the percentage of the illiterates in the Bhatia's standardization battery was almost equal to that of the literate sample. Further, the performance of the illiterate group was significantly lower compared to literate group. So, if one tries to merge both the norms, due to above mentioned reasons, the resultant set of norms would not be appropriate and will not represent the current abilities of the population. So, to answer the above question, that should both norms be merged, the humble answer is 'it is not a good idea'.

\section{Can this battery be administered to girls?}

The manual clearly mentions the limitations and the practical difficulties that it was impossible to recruit girls for intellectual assessment in 1940-50s in India.

Recent popular intelligence tests (Wechsler's tests, Raven's progressive matrices, and so on) do not have separate norms for the boys and girls. However, one of the important facts to remember is that these recent intelligence tests include 'females in standardization as one comprehensive sample of the population'. Therefore, when the females are included in the standardization sample, then the obtained norms can automatically be used irrespective of whether the person is a male or female.

However, Bhatia's battery has not included girls in the standardization sample. If girls would have been included in the standardization, probably the norms would have been different. In this regard, a restandardization study on Bhatia's battery (unknown authors) done in 1990s with about 500 subjects between the age of 11 years to 16 years ( 244 boys and 256 girls; 349 literates and 151 illiterates), showed that both boys and girls performed almost equally. Given this, with great hesitation and more than a pinch of salt, one can accept that the same norms can be used for both boys and girls. However, the better option would be to use other tests instead.

\section{Can it be administered for adults over 16 years?}

The battery norms are restricted to a narrow range in terms of the age, i.e. 11 to 16 years. However, some professionals do administer this battery on adults who are much older than 16 years. The maximum higher age it is administered varies across professionals, however, it is observed to be around 50 years. One of the possible arguments put forward by such professionals for its use in adults is that, intelligence rarely increases after 16 years, and hence the 16th year norms could be used for adults.

On some level, the argument relatively appeals to reason, because, other popularly used intelligence test, Binet Kamat Test of Intelligence [11-12] uses 16 years as the maximum chronological age while calculating the (ratio) IQ, despite the test has items to assess till 22 years. 
Though it is not the reason one site to administer it to adults. Bhatia battery itself provides an indirect reason that it is probably not wrong to use it on adults. The results of the standardization showed that the literate group relatively showed performance plateau at $15-16$ years, i.e. the scores for literate group did not show increasing pattern after 15 years (refer section 1.F. and figure 2). One can still argue that this plateau in performance might be a small aberration or a developmental trend; and if tested on higher ages, probably the performance of literates would have shown increasing tendency. However, this cannot be answered unless a new research is carried out using Bhatia for ages higher than 16 years.

Some of the other reasons, for some professionals using Bhatia's battery for adults, apparently include, that the battery is familiar and easy to use, it fosters interest and cooperation from the client, and provides correct IQ scores that seem to match the person's abilities. However, given a choice other intelligence tests that are recent, with appropriate representative standardization, and those with less limitations can be used. Further, they should be aware that the belief/perception that 'obtained IQ seem to match the person's abilities' is a subjective opinion and may or may not match the objective realities.

\section{Bhatia's Short form}

The administration of Bhatia's full battery takes about 45 minutes. Given the significant disparity in psychologists-to-patients' ratio, and especially where time is a limiting factor, test developers usually come up with short version of the full assessment versions. Similarly, psychologists have proposed a shorter version that includes Block Design and Pass Along subtests from Bhatia's battery [13-14]. Even though many use these two subtests as the short battery, there are two versions to arrive at full IQ. One, to sum up raw scores of Block Design and Pass Along subtests and multiply it with 2.5, and to compare with the overall-full norms [13]. Second, scores were allotted for each block of 30 seconds instead of 60 seconds as in Bhatia original method [14]. Though, multiplying with 2.5 version has found to be better than reducing the time-epoch method to get a full-scale IQ [15], it still not an appropriate and/or reliable method to obtain comprehensive IQ [15-16].

The mean average raw scores obtained in the standardization by Bhatia's [1] of each subtest is given in Table 3. It can be observed in this, that, though, the short-form method raw score (literate group $=44$; illiterate group $=26.3$ ) closely matches the total raw score (literate group $=46.45$; illiterate group 27.32), it is not the perfect match. One should remember that these raw scores of the standardization sample are the group mean/average scores. Group average scores tend to nullify the individual differences and/or extreme scores. Therefore, if in case, if one uses the short-form instead of the full battery on individuals who (have different specific abilities) perform differently on different tests, then they might get inappropriate IQ which does not match their actual ability (i.e. they might either get reduced or increased the full-scale IQ).

\section{The norms range only between 69 to 131 IQ points}

One of the major limitations usually talked about Bhatia's battery, is its apparently limited IQ range that it provides, which is only between 69 to 131 points. Usually intelligence tests that provide IQ as the final output value, always go beyond 69 to 131 IQ points on both sides, especially the lower side, where it is expected to indicate and differentiate the type of intellectual disability. This is not expected of those intelligence tests that provide the final output value in terms of percentile points, such as Raven progressive matrices.

One of the reasons why Bhatia's battery provides IQ points only between 69 to 131 is because, it is derived from (or arrived at after) extrapolating the percentile points. That is Bhatia's battery first calculated the 5th, 16th, 50th, 84th and 95th percentile points, and then fitted (through interpolation) the IQs to match the percentile points. It is inherent limitation of the percentile points that, at the extreme ends (more than \pm 2 SD) percentile points are difficult to differentiate (please refer Figure 5). Therefore, as Bhatia's battery adopted IQs from percentile points, the lowest and the highest points are 5th and 95th respectively, and percentile points does not provide much differentiation at the extreme ends the IQs are understandably limited between 69 to 131 . This can neither be a significant limitation nor an advantage. 
Table 3: Showing the difference between the total raw score (obtained by Bhatia [1]) and the short form raw score. The short form raw score followed the method introduced by Murthy [2].

\begin{tabular}{|c|c|c|}
\hline & $\begin{array}{c}\text { Literate group } \\
\text { Mean raw score }\end{array}$ & $\begin{array}{c}\text { Illiterate group } \\
\text { Mean raw score }\end{array}$ \\
\hline Block Design (BD) & 8.33 & 4.09 \\
\hline Pass Along (PA) & 9.27 & 6.43 \\
\hline Pattern Drawing & 9.0 & 5.18 \\
\hline Immediate Memory & 10.77 & 6.95 \\
\hline Picture Construction & 9.08 & 4.67 \\
\hline Total Raw Score & 46.45 & 27.32 \\
\hline BD \& PA X 2.5 & 44.0 & 26.3 \\
\hline
\end{tabular}

\section{CONCLUSIONS}

One of the major limitations of the Bhatia's battery is having a separate set of norms for literates and illiterates. Indeed, the researchers found significant differences between literates and illiterates, but does this alone warrants two separates norms? This 'significant difference between group result' is a research finding, it is a publication worthy finding, it is a finding that raises significant questions, debates and concerns about the role of literacy (or socioeconomic conditions, caste, and so on) in intelligence; and it is a finding that affects the policy decisions on a larger societal scale. But it is not the finding that requires separate set of norms.

One of the most important face that supports the argument that there is no requirement of separate set of norms, can be seen in Binet-Kamat test of intelligence [11-12]. BKT was standardized in 1920-30s by Kamat on Indian population in a small town in south India. This standardization predates about 2 decades in terms of time and given this, there should have been far more illiteracy among the population compared to 1040-50s when Bhatia's battery was standardized. However, BKT did not have any separate set of norms for literates and illiterates; nor did it use separate item-to-age-allotment criteria for literates and illiterates (BKT uses age scale). Further, according to this authors knowledge, there are no tests apart from Bhatia's battery that uses separate set of norms for literates and illiterates.

Another, important question one can ask is that, the standardization was done in 1950s, are the norms still be valid? This question generally applies to several of the tests that are developed in India. Most of the tests that are currently used in day-today practice by psychologists have been developed/standardized at least several decades back.

The one important aspect that warrants this question is the phenomenon of 'Flynn effect' [17]. The Flynn effect implies to the observation of increasing intelligence (over generations of people) of about 3 IQ points per decade. This phenomenon has been observed across the countries, however, the exact extent has varied among different countries over the different timeline [18].

India, presents a significant challenge with respect to intelligence assessment, due to it being a developing economy and a dual economy, where several super-rich and ultra-poor people exist in a same geographical location, with each group providing varied access to different levels of stimulation, opportunity, nutrition, interpersonal interaction and care to their children. In addition, India also has over 25 different official languages and further more dialects. All these factors might contribute to varying levels of Flynn effect, where for some group there can be more than 3 IQ points and for some groups there might be far less increase or no increase at all. Given this, it becomes difficult to say whether a battery which was standardized several decades back is still applicable now or not.

According to the publicly (online) available literature an attempt was made in 1990s to re-standardize the Bhatia's battery (unknown authors). The authors used 500 subjects between the age of 11 years to 16 years (244 boys and 256 girls; 349 literates and 151 illiterates). Even though the sample size was almost half of that of the original Bhatia's standardization sample and included both boys and girls, the authors found that the IQ of the groups (both illiterate and literate groups) was only marginally increased. However, this 
study is not without its limitations, such as they still retained the literates and illiterates as separate groups. Further, according to the available knowledge, it is not sure whether this study has been published in any peer reviewed journal or manuscript.

However, some psychologists use this battery mainly to confirm their belief or clinical judgment (based on the feedback from the family, or their interaction with the person) that the person's IQ is around 'normal' range to rule out any issues of mental retardation/intellectual disability. Further, the battery is used in clinical setting to determine whether the patient can understand treatment demands and/or to start some form of psychotherapy that require patient to be able to understand and reflect on the psychotherapy process. If it is used in such a scenario then, using this battery rarely affects the person adversely.

Therefore, the answer to the question that 'is the battery still be valid after decades old standardization?', the answer is, it depends on 'why' and 'on whom' it is used. It is not advisable to use for school and career guidance, definitely not for disability assessment and legal issues. Further, it is not advisable to use it for illiterates, even with the separate norms for illiterates (refer sections 1.A. to 1.M.). In addition to the reason that it provides IQs only from 69 to 131, it is not advisable to use it for those who are suspected to have extreme levels (on either side of the NPC) of intelligence.

\section{REFERENCES}

1. Bhatia CM. Performance Tests of Intelligence Under Indian conditions. London: Oxford University Press; 1955.

2. Becker KA. History of the Stanford-Binet intelligence scales: Content and psychometrics (Stanford-Binet Intelligence Scales, Fifth Edition Assessment Service Bulletin No. 1). Ilinois: Riverside Publishing; 2003.

3. Wechsler D. Wechsler Intelligence Scale for Children. 4th ed. Texas: Harcourt Assessment; 2003.

4. World Health Organization. International Classification of Diseases and Related Health Problems. 10th ed; 1992.

5. UNESCO. The Race concept. Paris: UNESCO, 1952.

6. Ritchie SJ, Tucker-Drob EM. How Much Does Education Improve Intelligence? A Meta-Analysis. Psychol Sci 2018;29:1358-69.

7. Hoosain R, Salili F. Language differences in pronunciation speed for numbers, digit span, and mathematical ability. Psychologia 1987;30:34-8.

8. Elliott JM. Forward digit span and articulation speed for Malay, English, and two Chinese dialects. Percept Motor Skills 1992;74:291-5.

9. Chan ME, Elliott JM. Cross-Linguistic Differences in Digit Memory Span. Austral Psychol 2011;46:25-30.

10. World Health Organization. International Classification of Functioning, Disability and Health (ICF). Geneva: 2001, WHO.

11. Kamat VV. Measuring Intelligence of Indian children. 1st ed. Bombay: Oxford University Press; 1934.

12. Kamat VV. Measuring intelligence of Indian children. 3rd ed. Bombay: Oxford University Press; 1967.

13. Murthy HN. A short scale of the Bhatia's performance tests. Indian Psycho Rev 1966;2:133-4.

14. Pershad D, Mahajan A, Verma SK. Bhatia's short scale of intelligence - A suggested modification for adults. Personal Stud Group Behav 1988; 8:43-8.

15. Verma SK, Pershad D, Malhotra A, Arunima M. The Revised Bhatia's Short Battery of performance Tests of Intelligence for Adults - A Hand Book. Agra: National psychological Corporation; 1988.

16. Barnabas IP. Comparison of two Short Forms of the Bhatia's test of Intelligence. NIMHANS Journal, 1994; 12;75-77.

17. Cronbach LJ. Essential of Psychological Testing. 4th ed. New York: Haper \& Row; 1984.

18. Flynn JR. The mean IQ of Americans: Massive gains 1932 to 1978. Psychol Bull 1984;95:29-51.

19. Kaufman AS. IQ Testing 101. USA: Springer; 2009.

$* * * * * * * * * * * * * * * * * * * * * * * * * * * * * * * * * * * *$

Acknowledgements - Nil

Conflict of Interest - Nil

Funding - Nil 